\title{
Theory of Superconductivity in Graphite Intercalation Compounds
}

\author{
Yasutami Takada ${ }^{1, *}$ \\ ${ }^{1}$ Institute for Solid State Physics, University of Tokyo, \\ 5-1-5 Kashiwanoha, Kashiwa, Chiba 277-8581, Japan
}

\begin{abstract}
On the basis of the model that was successfully applied to $\mathrm{KC}_{8}, \mathrm{RbC}_{8}$, and $\mathrm{CsC}_{8}$ in 1982 , we have calculated the superconducting transition temperature $T_{c}$ for $\mathrm{CaC}_{6}$ and $\mathrm{YbC}_{6}$ to find that the same model reproduces the observed $T_{c}$ in those compounds as well, indicating that it is a standard model for superconductivity in the graphite intercalation compounds with $T_{c}$ ranging over three orders of magnitude. Further enhancement of $T_{c}$ well beyond $10 \mathrm{~K}$ is also predicted. The present method for calculating $T_{c}$ from first principles is compared with that in the density functional theory for superconductors, with paying attention to the feature of determining $T_{c}$ without resort to the concept of the Coulomb pseudopotential $\mu^{*}$.
\end{abstract}

PACS numbers: 74.70.Wz,74.20.-z,74.20.Pq

\section{INTRODUCTION}

\section{A. Crystal Structure}

For many decades, graphite intercalation compounds (GICs) have been investigated from the viewpoints of physics, chemistry, materials science, and engineering (or technological) applications $\underline{\underline{1}} \underline{\underline{4}}$. Among various kinds of GICs, special attention has been paid to the first-stage metal compounds, partly because superconductivity is observed mostly in this class of GICs, the chemical formula of which is written as $M \mathrm{C}_{x}$, where $M$ represents either an alkali atom (such as $\mathrm{Li}, \mathrm{K}, \mathrm{Rb}$, and $\mathrm{Cs}$ ) or an alkaline-earth atom (such as $\mathrm{Ca}, \mathrm{Sr}, \mathrm{Ba}$, and $\mathrm{Yb}$ ) and $x$ is either 2,6 , or 8 . The crystal structure of $M \mathrm{C}_{x}$ is shown in Fig. 1(a), in which the metal atom $M$ occupies the same spot in the framework of a honeycomb lattice at every $(x / 2)$ layers of carbon atoms.

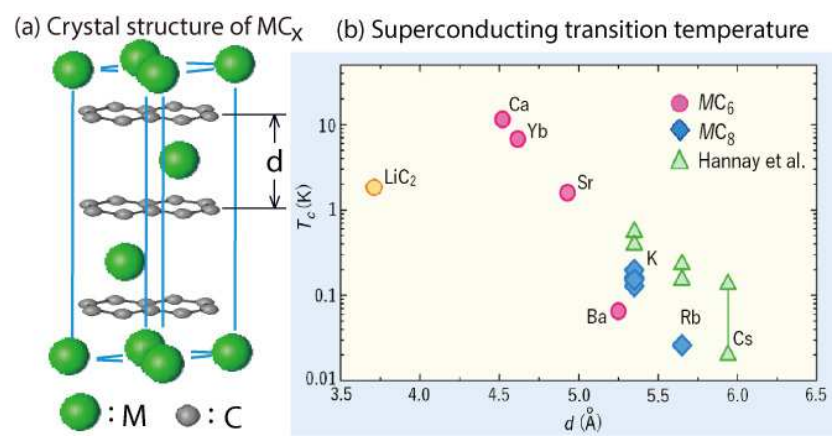

FIG. 1: (a) Crystal structure of $\left.M \mathrm{C}_{x} \mathrm{i} x=2,6,8\right)$. The case of $x=6$ is illustrated here, in which the metal atoms, $M \mathrm{~s}$, are arranged in a rhombohedral structure with the $\alpha \beta \gamma$ stacking sequence, implying that $M$ occupies the same spot in the framework of a graphene lattice at every three layers (or at the distance of $3 d$ with $d$ the distance between the adjacent graphite layers). (b) Superconducting transition temperature $T_{c}$ observed in the first-stage alkali- and alkaline-earth-intercalated graphites plotted as a function of $d$.

\section{B. Superconductivity}

The first discovery of superconductivity in GICs was made in $\mathrm{KC}_{8}$ with the superconducting transition temperature $T_{c}$ of $0.15 \mathrm{~K}$ in $1965^{\frac{5}{}}$. In pursuit of higher $T_{c}$, various GICs were synthesized, mostly working with the alkali metals and alkali-metal amalgams as intercalants, from the late 1970 s to the early $1990 \mathrm{~s}^{\underline{6}-13}$, but only a limited success was achieved at that time; the highest attained $T_{c}$ was around $2-5 \mathrm{~K}$ in the last century. For example, it is $1.9 \mathrm{~K}$ in $\mathrm{LiC}_{2} \underline{14}$.

A breakthough occurred in 2005 when $T_{c}$ went up to $11.5 \mathrm{~K}$ in $\mathrm{CaC}_{6} \frac{15.16}{}$ (and even to $15.4 \mathrm{~K}$ under pressures up to $7.5 \mathrm{GPa} 17$ ). In other alkaline-earth GICs, the values of $T_{c}$ are $6.5 \mathrm{~K}, 1.65 \mathrm{~K}$, and $0.065 \mathrm{~K}$ for $\mathrm{YbC}_{6}{ }^{15}, \mathrm{SrC}_{6}{ }^{18}$, and $\mathrm{BaC}_{6} \frac{19}{9}$, respectively, as indicated in Fig. 1(b). Since then, very intensive experimental studies have been made in those and related compounds $16,18,20,21$. Theoretical studies have also been performed mainly by making state-of-the-art first-principles calculations of the electron-phonon coupling constant $\lambda$ to account for the observed value of $T_{c}$ for each individual superconductor $^{22}$-26. Those experimental/theoretical works have elucidated that, although there are some anisotropic features in the superconducting gap, the conventional phonon-driven mechanism to bring about s-wave superconductivity applies to those compounds. This picture of superconductivity is confirmed by, for example, the observation of the Ca isotope effect with its exponent $\alpha=0.50$, the typical Bardeen-Cooper-Schrieffer (BCS) value 27 .

\section{Central Issues}

In spite of all those efforts and the existence of such a generally accepted picture, there remain several very important and fundamental questions:

1. Can we understand the mechanism of superconductivity in both alkali GICs with $T_{c}$ in the range $0.01-1.0 \mathrm{~K}$ and alkaline-earth GICs with $T_{c}$ typi- 
cally in the range $1-10 \mathrm{~K}$ from a unified point of view? In other words, is there any standard model for superconductivity in GICs with $T_{c}$ ranging over three orders of magnitude?

2. What is the actual reason why $T_{c}$ is enhanced so abruptly (or by about a hundred times) by just substituting $\mathrm{K}$ by $\mathrm{Ca}$ the atomic mass of which is almost the same as that of K? In terms of the standard model, what are the key controlling physical parameters to bring about this huge enhancement of $T_{c}$ ? This change of $T_{c}$ from $\mathrm{KC}_{8}$ to $\mathrm{CaC}_{6}$ is probably the most important issue in exploring superconductivity across the entire family of GICs.

3. Is there any possibility to make a further enhancement of $T_{c}$ in GICs? If possible, what is the optimum value of $T_{c}$ expected in the standard model and what kind of atoms should be intercalated to realize the optimum $T_{c}$ in actual GICs?

4. What is the physical reason why $\mathrm{BaC}_{6}$ provides so low $T_{c}(=0.065 \mathrm{~K})$ experimentally, compared with other alkaline-earth GICs and also with $T_{c}=0.23 \mathrm{~K}$ in the conventional Eliashberg theory ${ }^{25}$ ? This issue may not be important in obtaining high- $T_{c}$ superconductors, but physically it is important enough in comprehensively understanding the mechanism of superconductivity in GICs.

In order to provide reliable answers to the above questions, it is indispensable to make a first-principles calculation of $T_{c}$ with sufficient accuracy and predictive power. Several years ago, such a calculation was completed by the present author and on the basis of the calculation, some interesting predictions has been pro$\operatorname{posed}^{28,29}$. The present chapter not only reports some details of this work on the superconducting mechanism in GICs but also makes a brief summary of the current status of the theories for first-principles calculations of $T_{c}$.

\section{Organization of This Chapter}

This chapter is organized as follows: In Secs. 2-4, a critical review of the theories for quantitative calculations of $T_{c}$ is given. More specifically, we make comments on the theories based on the McMillan's or the Allen-Dynes' formula employing the concept of the Coulomb pseudopotential $\mu^{*}$ in Sec. 2. In Sec. 3 we explain the theory on the level of the so-called $G_{0} W_{0}$ approximation, in which it is a very important merit that $T_{c}$ can be obtained without using $\mu^{*}$. The same merit can be enjoyed in the density functional theory for superconductors, which will be addressed in Sec. 4. In Secs. 5-8, a review on superconductivity in GICs is given; starting with a summary of the experimental works in Sec. 5, a standard model for considering the mechanism of superconductivity in GICs is introduced in Sec. 6. In Sec. 7, the calculated results of $T_{c}$ are given for the alkaline-earth GICs and they are compared with the experimental results. The prediction of the optimum $T_{c}$ is given in Sec. 8. Finally in Sec. 9, the conclusion of this chapter is given, together with some perspectives on the researches in this and related fields in the future.

\section{FIRST-PRINCIPLES CALCULATION OF $T_{c}$}

\section{A. Goal of the Problem}

It would be one of the ultimate goals in the enterprise of condensed matter theory to make a reliable prediction of $T_{c}$ only through the information on constituent elements of a superconductor in consideration. A less ambitious yet very important goal is to make an accurate evaluation of $T_{c}$ directly from a microscopic (model) Hamiltonian pertinent to the superconductor. If we could find the dependence of $T_{c}$ on the parameters specifying the model Hamiltonian, we could obtain a deep insight into the mechanism of superconductivity or the competition between the attractive and the repulsive interactions between electrons. Accumulation of such information might pave the way to the synthesis of a room-temperature superconductor, a big dream in materials science. From this perspective, a continuous effort has been made for a long time to develop a good theory for first-principles calculations of $T_{c}$, starting with a microscopic Hamiltonian.

\section{B. McMillan's and Allen-Dynes' Formulas for $T_{c}$}

In the phonon mechanism, for example, there has been a rather successful framework for this purpose, known as the McMillan's formula ${ }^{30}$ or its revised version (the Allen-Dynes' formula) ${ }^{31-33}$, both of which are derived from the Eliashberg theory of superconductivity ${ }^{34}$. In this framework, the task of a microscopic calculation of $T_{c}$ is reduced to the evaluation of the so-called Eliashberg function $\alpha^{2} F(\omega)$ from first principles; the function $\alpha^{2} F(\omega)$ enables us to obtain both the electron-phonon coupling constant $\lambda$ and the average phonon energy $\omega_{0}$, through which we can make a first-principles prediction of $T_{c}$ with an additional introduction of a phenomenological parameter $\mu^{*}$ (the Coulomb pseudopotential ${ }^{35}$ ) in order to roughly estimate the effect of the Coulomb repulsion between electrons on $T_{c}$.

At present this framework is usually regarded as the canonical one for making a first-principles prediction of $T_{c}$. In fact, the superconducting mechanism of many (so-called weakly-correlated) superconductors is believed to be clarified by using this framework, whereby the key phonon modes to bring about superconductivity are identified. We can point out that superconductivity in $\mathrm{MgB}_{2}$ 
with $T_{c}=39 \mathrm{~K}$ is a good recent example ${ }^{36}-\underline{39}$ to illustrate the power of this framework. The case of $\mathrm{CaC}_{6}$ has also been investigated along this line of theoretical studies 22,24 .

\section{Coulomb Pseudopotential $\mu^{*}$}

Nevertheless, this framework is not considered to be very satisfactory, primarily because a phenomenological parameter $\mu^{*}$ is included in the theory. Actually, it cannot be regarded as the method of predicting $T_{c}$ in the true sense of the word, if the parameter $\mu^{*}$ is determined so as to reproduce the observed $T_{c}$. Besides, as long as we employ $\mu^{*}$ to avoid a serious investigation of the effects of the Coulomb repulsion on superconductivity, we cannot apply this framework to strongly-correlated superconductors. Even in weakly- or moderately-correlated superconductors, this framework cannot tell anything about superconductivity originating from the Coulomb repulsion via charge and/or spin fluctuations (namely, the electronic mechanism including the plasmon mechanism $\left.{ }^{40,41}\right)$. Furthermore, in this framework, we cannot investigate the competition or the coexistence (or even the mutual enhancement due to the quantum-mechanical interference effect) between the phonon and the electronic mechanisms.

The validity of the concept of $\mu^{*}$ is closely connected with that of the Eliashberg theory itself; the theory is valid only when the Fermi energy of the superconducting electronic system, $E_{\mathrm{F}}$, is very much larger than $\omega_{0}$. Under the condition of $E_{\mathrm{F}} \gg \omega_{0}$, the dynamic response time for the phonon-mediated attraction $\omega_{0}^{-1}$ is much slower than that for the Coulomb repulsion $E_{\mathrm{F}}^{-1}$, precluding any possible interference effects between two interactions, so that physically it is very reasonable to separate them. After this separation, the Coulomb part (which was not considered to play a positive role in the Cooper-pair formation) has been simply treated in terms of $\mu^{*}$. Thus, for the purpose of searching for some positive role of the Coulomb repulsion, the concept of $\mu^{*}$ is irrelevant from the outset.

\section{Vertex Corrections and Dynamic Screening}

Incidentally, in some recently discovered superconductors in the phonon mechanism such as the alkali-doped fullerenes with $T_{c}=18-38 \mathrm{~K}^{42} \underline{45}$, the condition of $E_{\mathrm{F}} \gg \omega_{0}$ is violated, necessitating to include the vertex corrections in calculating the phonon-mediated attractive interaction ${ }^{46}$. Then, it is by no means clear to treat the overall effect of various phonons in terms of the sum of the contribution from each phonon, implying that the Eliashberg function $\alpha^{2} F(\omega)$ is not enough to properly describe the attraction because of possible interference effects among virtually-excited phonons. As a consequence, $\lambda$ will not be simply the sum of $\lambda_{i}$ the con- tribution from the $i$ th phonon, unless $\lambda_{i}$ is small enough to validate the whole calculation in lowest-order perturbation.

In the case of $E_{\mathrm{F}} \approx \omega_{0}$, another complication occurs in treating the screening effect of the conduction electrons. In the usual first-principles calculation scheme, the static screening is assumed in calculating $\alpha^{2} F(\omega)$, but it does not reflect the actual screening process working during the formation of Cooper pairs.

\section{E. Ideal Calculation Scheme}

In order to unambiguously solve this problem of screening, we may imagine a following ideal calculation scheme for $T_{c}$ : In the first step, we calculate the microscopic dynamical electron-electron effective interaction $V$ in the whole momentum and energy space. This $V$ is assumed to contain both the Coulomb repulsion and the phononmediated attraction on the same footing. Then in the second step, we obtain $T_{c}$ directly from this $V$ with simultaneously determining the gap function in the whole momentum and energy space, reflecting the behavior of $V$. If this scheme were developed, we could not only calculate $T_{c}$ from first principles without resort to $\mu^{*}$ but also correctly discuss the competition, coexistence, and mutual enhancement between the phonon and the electronic mechanisms.

\section{CALCULATION OF $T_{c}$ IN THE $G_{0} W_{0}$ APPROXIMATION}

\section{A. Formulation}

Although it is along the royal road in the project of obtaining a reliable method for predicting $T_{c}$ from first principles, this ideal calculation scheme is extremely difficult to achieve in actual situations, because all the difficulties in the quantum-mechanical many-body problem are associated with it. About three decades ago, the present author, who was a graduate student at that time, was struggling with developing such a scheme without perceiving much of the difficulties intrinsic to the many-body problem. After a year-long struggle, he managed to propose a rather general scheme to evaluate $T_{c}$ directly from $V$ without introducing the concept of $\mu^{*} \underline{\underline{40}}$, though it was still at the stage far from the ideal scheme.

In a broad sense, this scheme may be called an approach from the weak-coupling limit, corresponding to the $G_{0} W_{0}$ approximation or the one-shot $G W$ approximation in the terminology of the present-day firstprinciples calculation community. In the same terminology, by the way, the Eliashberg theory corresponds to the $G W$ approximation with respect to the phonon-mediated attractive interaction between electrons.

Let us explain this $G_{0} W_{0}$ scheme here $\underline{\underline{47}}$. For simplicity, imagine the three-dimensional (3D) electron gas 
in which an electron is specified by momentum $\mathbf{p}$ and spin $\sigma$. If we write the electron annihilation operator by $c_{\mathbf{p} \sigma}$, we can define the abnormal thermal Green's function $F\left(\mathbf{p}, i \omega_{p}\right)$ at temperature $T$ by

$$
F\left(\boldsymbol{p}, i \omega_{p}\right)=-\int_{0}^{1 / T} d \tau e^{i \omega_{p} \tau}\left\langle T_{\tau} c_{\boldsymbol{p} \uparrow}(\tau) c_{-\boldsymbol{p} \downarrow}\right\rangle
$$

with $\omega_{p}$ the fermion Matsubara frequency. At $T=T_{c}$ where the second-order phase transition occurs, this function satisfies the following exact gap equation:

$$
\begin{aligned}
F\left(\boldsymbol{p}, i \omega_{p}\right) & =-G\left(\boldsymbol{p}, i \omega_{p}\right) G\left(-\boldsymbol{p},-i \omega_{p}\right) \\
& \times T_{c} \sum_{\omega_{p^{\prime}}} \sum_{\boldsymbol{p}^{\prime}} \tilde{I}\left(\boldsymbol{p}, \boldsymbol{p}^{\prime} ; i \omega_{p}, i \omega_{p^{\prime}}\right) F\left(\boldsymbol{p}^{\prime}, i \omega_{p^{\prime}}\right),
\end{aligned}
$$

where $G\left(\boldsymbol{p}, i \omega_{p}\right)$ is the normal Green's function and $\tilde{I}\left(\boldsymbol{p}, \boldsymbol{p}^{\prime} ; i \omega_{p}, i \omega_{p^{\prime}}\right)$ is the irreducible electron-electron effective interaction.

Now, in the spirit of the $G_{0} W_{0}$ approximation, we replace $G\left(\boldsymbol{p}, i \omega_{p}\right)$ by the bare one $G_{0}\left(\boldsymbol{p}, i \omega_{p}\right) \equiv\left(i \omega_{p}-\varepsilon_{\boldsymbol{p}}\right)^{-1}$ in Eq. (2), where $\varepsilon_{\boldsymbol{p}}\left(=\boldsymbol{p}^{2} / 2 m^{*}-\mu\right)$ is the bare oneelectron dispersion relation with $m^{*}$ the band mass and $\mu$ the chemical potential. We will also consider the case in which $\tilde{I}\left(\boldsymbol{p}, \boldsymbol{p}^{\prime} ; i \omega_{p}, i \omega_{p^{\prime}}\right)$ is well approximated as a function of only the variables $\left(\boldsymbol{p}-\boldsymbol{p}^{\prime}, i \omega_{p}-i \omega_{p^{\prime}}\right)$ to write

$$
\tilde{I}\left(\boldsymbol{p}, \boldsymbol{p}^{\prime} ; i \omega_{p}, i \omega_{p^{\prime}}\right)=V\left(\boldsymbol{p}-\boldsymbol{p}^{\prime}, i \omega_{p}-i \omega_{p^{\prime}}\right)
$$

just like the effective interaction in the random-phase approximation (RPA), though we do not intend to confine ourselves to the RPA at this point. By substituting Eq. (3) into Eq. (2) and making an analytic continuation on the $\omega$ plane to transform $F\left(\boldsymbol{p}, i \omega_{p}\right)$ to the retarded function $F^{R}(\boldsymbol{p}, \omega)$ on the real- $\omega$ axis, we get a gap equation for $F^{R}(\boldsymbol{p}, \omega)$. Then, by taking the imaginary parts in both sides of the gap equation and integrating over the $\omega$ variable, we finally obtain an equation depending only on the momentum variable $\boldsymbol{p}$. Concretely, the equation can be cast into the following BCS-type gap equation:

$$
\Delta_{\boldsymbol{p}}=-\sum_{\boldsymbol{p}^{\prime}} \frac{\Delta_{\boldsymbol{p}^{\prime}}}{2 \varepsilon_{\boldsymbol{p}^{\prime}}} \tanh \frac{\varepsilon_{\boldsymbol{p}^{\prime}}}{2 T_{c}} \mathcal{K}_{\boldsymbol{p}, \boldsymbol{p}^{\prime}}
$$

where the gap function $\Delta_{p}$ and the pairing interaction $\mathcal{K}_{\boldsymbol{p}, \boldsymbol{p}^{\prime}}$ are, respectively, defined by

$$
\Delta_{\boldsymbol{p}} \equiv 2\left|\varepsilon_{\boldsymbol{p}}\right| \int_{0}^{\infty} \frac{d \omega}{\pi} \operatorname{Im} F^{R}(\boldsymbol{p}, \omega)
$$

and

$$
\mathcal{K}_{\boldsymbol{p}, \boldsymbol{p}^{\prime}}=\int_{0}^{\infty} \frac{2}{\pi} d \Omega \frac{\left|\varepsilon_{\boldsymbol{p}}\right|+\left|\varepsilon_{\boldsymbol{p}^{\prime}}\right|}{\Omega^{2}+\left(\left|\varepsilon_{\boldsymbol{p}}\right|+\left|\varepsilon_{\boldsymbol{p}^{\prime}}\right|\right)^{2}} V\left(\boldsymbol{p}-\boldsymbol{p}^{\prime}, i \Omega\right) .
$$

With use of $\mathcal{K}_{\boldsymbol{p}, \boldsymbol{p}^{\prime}}$ thus calculated, we can determine $T_{c}$ as an eigenvalue of Eq. (4).

\section{B. Comments on the Formulation}

Five comments are in order on this framework:

i) Based on Eqs. (4) and (6), we can obtain $T_{c}$ directly from the microscopic one-electron dispersion relation $\varepsilon_{\boldsymbol{p}}$ and the effective electron-electron interaction $V(\boldsymbol{q}, i \Omega)$ with no need to separate the phononmediated attraction from the Coulomb repulsion.

ii) In spite of the similarity of Eq. (4) to the BCS gap equation, artificial cutoffs involved in constructing the BCS model are avoided in the present scheme; natural cutoffs are automatically introduced by the calculation of $\mathcal{K}_{\boldsymbol{p}, \boldsymbol{p}^{\prime}}$ defined in Eq. (6).

iii) Except for the spin-singlet pairing, no assumption is made on the dependence of the gap function on angular valuables in deriving Eq. (44), so that this gap equation can treat any kind of anisotropy in the gap function, indicating that it can be applied to s-wave, d-wave, $\cdots$, and even their mixture like $(\mathrm{s}+\mathrm{d})$-wave superconductors.

iv) As can be seen by its definition, the gap function $\Delta_{p}$ in Eq. (5) does not correspond to the physical energy gap except in the weak-coupling region. Similary, $\mathcal{K}_{\boldsymbol{p}, \boldsymbol{p}^{\prime}}$ is not a physical entity. Both quantities are introduced for the mathematical convenience so as to make $T_{c}$ invariant in transforming Eq. (2) into Eq. (4). The key point here is that we need not solve the full gap equation (2) but much simpler one (4) in order to obtain $T_{c}$ in Eq. (2). Of course, if we want to know the physical gap function rather than $\Delta_{p}$ to compare with experiment, we need to solve the full gap equation, Eq. (2), with $T_{c}$ determined by Eq. (41).

v) Historically, Cohen was the first to evaluate $T_{c}$ in degenerate semiconductors on the level of the $G_{0} W_{0}$ approximation ${ }^{48,49}$. Unfortunately the pairing interaction is not correctly derived in his theory, as explicitly pointed out by the present author 50 who, instead, has succeeded in obtaining the correct pairing interaction 40 by consulting the pertinent work of Kirzhnits et al. 51 .

\section{Assessment: Application to $\mathrm{SrTiO}_{3}$}

In order to assess the quality of this basic framework of calculating $T_{c}$ from first principles, we have applied it to $\mathrm{SrTiO}_{3}$ and compared the results with experiments ${ }^{50}$. This material is an insulator and exhibits ferroelectricity under a uniaxial stress of about 1.6kbar along the [100] direction, but it turns into an $n$-type semiconductor by either $\mathrm{Nb}$ doping or oxygen deficiency, whereby the conduction electrons are introduced in the $3 \mathrm{~d}$ band of $\mathrm{Ti}$ around the $\Gamma$ point with the band mass of $m^{*} \approx 1.8 m_{e}$ $\left(m_{e}\right.$ : the mass of a free electron). At low temperatures, 
superconductivity appears and the observed $T_{c}$ shows interesting features; $T_{c}$ depends strongly on the electron concentration $n$ and it is optimized with $T_{c} \approx 0.3 \mathrm{~K}$ at $n \approx 10^{20} \mathrm{~cm}^{-3}$. Its dependence on the pressure is unsual; $T_{c}$ decreases rather rapidly with hydrostatic pressures, but it increases with the [100] uniaxial stress.
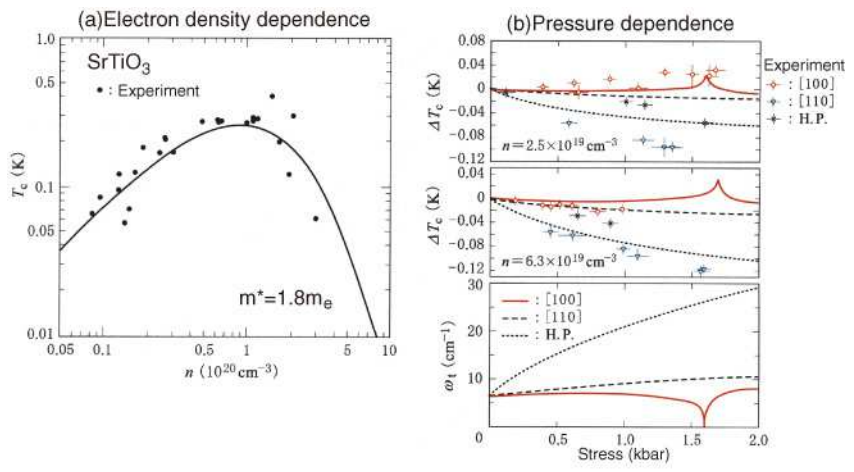

FIG. 2: (a) Electron density dependence of $T_{c}$ in $\mathrm{SrTiO}_{3}$ and (b) pressure dependence of $T_{c}^{50}$. Both uniaxial stress along either [100] or [110] direction and hydrostatic pressure (H.P.) are considered. The deviation of $T_{c}, \Delta T_{c}$, is plotted as a function of stress in units of kbar. For comparison, corresponding experimental results are also shown, together with the results of the transverse polar phonon energy $\omega_{t}$ in units of $\mathrm{cm}^{-1}$.

Taking those situations into account, we have assumed that the superconductivity is brought about by the polarcoupling phonons associated with the stress-induced ferroelectric phase transition. Then we have calculated the effective electron-electron interaction $V(\boldsymbol{q}, i \Omega)$ in the RPA, in which the long-range attraction induced by the virtual exchange of polar-coupled phonons is included with the long-range Coulomb repulsion on the same footing. By substituting this $V(\boldsymbol{q}, i \Omega)$ into Eq. (6), we have obtained $T_{c}$ directly from a microscopic model and the results of $T_{c}$ are in surprizingly good quantitative agreement with experiment, as shown in Fig. 2. Recently, a further experimental study on superconductivity in this electron-doped $\mathrm{SrTiO}_{3}$ was made to confirm the above results, together with the value for the effective mass of $m^{*}=(1.82 \pm 0.05) m_{e} e^{52,53}$. This success indicates that the present basic framework including the adoption of the RPA is very useful at least in the polar-coupled phonon mechanism.

\section{DENSITY FUNCTIONAL THEORY FOR SUPERCONDUCTORS}

\section{A. Hohenberg-Kohn-Sham Theorem}

Recently much attention has been paid to an extension of the density functional theory (DFT) to treat superconductivity, mainly because it provides another scheme for first-principles calculations of $T_{c}$ without resort to $\mu^{*}$. We shall make a very brief review of it in this section.
It is stated in the basic theorem in DFT that all the physics of an interacting electron sysytem is uniquely determined, once its electronic density in the ground state $n(\boldsymbol{r})$ is specified. This Hohenberg-Kohn theorem ${ }^{54} \mathrm{im}-$ plies that every physical quantity including the exchangecorrelation energy $F_{x c}$ may be considered as a unique functional of $n(\boldsymbol{r})$. The density $n(\boldsymbol{r})$ itself can be determined by solving the ground-state electronic density of the corresponding noninteracting reference system that is stipulated in terms of the Kohn-Sham (KS) equation ${ }^{55}$. The core quantity in the KS equation is the exchange-correlation potential $V_{x c}(\boldsymbol{r})$, which is defined as the functional derivative of $F_{x c}[n(\boldsymbol{r})]$ with respect to $n(\boldsymbol{r})$, namely, $V_{x c}(\boldsymbol{r})=\delta F_{x c}[n] / \delta n(\boldsymbol{r})$. It must be noted that $V_{x c}(\boldsymbol{r})$ as well as each one-electronic wavefunction at $i$ th level with its energy eigenvalue $\varepsilon_{i}$ in the KS equation has no physical relevance; they are merely introduced for the mathematical convenience so as to obtain the exact $n(\boldsymbol{r})$ in connecting the noninteracting reference system with the real many-electron system.

The Hohenberg-Kohn theorem can be applied to the ordered ground state as well on the understanding that the order parameter itself is regarded as a functional of $n(\boldsymbol{r})$. In providing some approximate functional form for $F_{x c}[n]$, however, it would be more convenient to treat the order parameter as an additional independent variable. For example, in considering the system with some magnetic order, we usually employ the spin-dependent scheme in which the fundamental variable is not $n(\boldsymbol{r})$ but the spin-decomposed density $n_{\sigma}(\boldsymbol{r})$, leading to the spinpolarized exchange-correlation energy functional $F_{x c}\left[n_{\sigma}\right]$, based on which the spin-dependent KS equation is formulated.

\section{B. Gap Equation}

Similarly, in treating the superconducting state in the framework of DFT, it is better to construct the energy functional with employing both $n(\boldsymbol{r})$ and the electronpair density $\chi\left(\boldsymbol{r}, \boldsymbol{r}^{\prime}\right)\left(\equiv\left\langle\Psi_{\uparrow}(\boldsymbol{r}) \Psi_{\downarrow}\left(\boldsymbol{r}^{\prime}\right)\right\rangle\right)$ as basic variables, leading to the introduction of the exchange-correlation energy functional $F_{x c}\left[n(\boldsymbol{r}), \chi\left(\boldsymbol{r}, \boldsymbol{r}^{\prime}\right)\right]$, where $\Psi_{\sigma}(\boldsymbol{r})$ is the electron annihilation operator ${ }^{56,57}$. In accordance with this addition of the order parameter as a fundamental variable to DFT, not only the exchange-correlation potential $V_{x c}(\boldsymbol{r})$ but also the exchange-correlation pairpotential $\Delta_{x c}\left(\boldsymbol{r}, \boldsymbol{r}^{\prime}\right)=-\delta F_{x c}[n, \chi] / \delta \chi^{*}\left(\boldsymbol{r}, \boldsymbol{r}^{\prime}\right)$ appear in an extended KS equation, which is found to be written in the form of the Bogoliubov-de Gennes equation appearing in the usual theory for inhomogeneous superconductors $\frac{58}{}$. Just as is the case with $V_{x c}(\boldsymbol{r}), \Delta_{x c}\left(\boldsymbol{r}, \boldsymbol{r}^{\prime}\right)$ has no direct physical meaning, but in principle, if the exact form of $F_{x c}[n, \chi]$ is known, the solution of the extended KS equation gives us the exact result for $\chi\left(\boldsymbol{r}, \boldsymbol{r}^{\prime}\right)$, containing all the effects of the Coulomb repulsion including the one usually treated phenomenologically through the concept of $\mu^{*}$. As a result, we can determine the exact 
$T_{c}$ by the calculation of the highest temperature below which a nonzero solution for $\chi\left(\boldsymbol{r}, \boldsymbol{r}^{\prime}\right)$ can be found.

In this formulation, we can write the fundamental gap equation to determine $T_{c}$ exactly as

$$
\Delta_{i}=-\sum_{j} \frac{\Delta_{j}}{2 \varepsilon_{j}} \tanh \frac{\varepsilon_{j}}{2 T_{c}} \mathcal{K}_{i j}
$$

where $\Delta_{i}$ is the gap function for $i$ th KS level. In just the same way as its energy eigenvalue $\varepsilon_{i}$ (which is measured from the chemical potential), $\Delta_{i}$ is not the quantity to be observed experimentally but just introduced for the mathematical convenience so as to obtain the exact $T_{c}$ by solving this BCS-type equation, Eq. (7). Similarly, the pair interaction $\mathcal{K}_{i j}$, defined as the second-functional derivative of $F_{x c}[n, \chi]$ with respect to $\chi^{*}$ and $\chi$, has not any direct physical meaning, either.

We note here the very impressive fact that the final forms for the two gap equations, Eqs. (4) and (7), are exactly the same, in spite of the fact that they are derived from quite different foundations and reasoning. We also note that because of this similarity, we may judge that, as long as $\mathcal{K}_{i j}$ is properly chosen, the physics descibed by $\mu^{*}$ is also included in the framework of DFT for superconductors, at least to the extent that it is included in the $G_{0} W_{0}$ scheme explained in Sec. 3 .

\section{Applications}

In 2005, this DFT framework was extended to explicitly taking care of the phonon-mediated attractive interaction ${ }^{59}$ and it has been applied to many superconductors $26,60-64$. In order to perform these calculations for actual superconductors, it is necessary to provide a concrete form for $F_{x c}[n, \chi]$. In the judgement of the present author, the presently available form for $F_{x c}[n, \chi]$ contains the information equivalent to that included in the Eliashberg theory for the part of the phonon-mediated attraction, indicating that no vertex corrections are considered in this treatment, while for the part of the Coulomb repulsion, it contains only very crude physics; the screening effect is treated in the Thomas-Fermi static-screening approximation, which is nothing but the result of the RPA only in the static and the long-wavelength limit, forgetting the detailed dynamical nature of the screening effect. Mainly for this reason, $T_{c}$ in the present form of $F_{x c}[n, \chi]$ is not expected to be very accurate, even though the calculated results for $T_{c}$ seem to be in good agreement with expeiment.

\section{Basic Problems}

In relation to the above point, it would be appropriate to give a following comment: In the calculations of the normal-state properties in the local-density approximation (LDA) and generalized gradient approximation
(GGA) $)^{65}$ to DFT, we usually anticipate that errors in the calculated results are of the order of $1 \mathrm{eV}$ and $0.3 \mathrm{eV}$ for LDA and GGA, respectively. Those errors are much larger than that expected in the calculation of quantum chemistry $(\approx 0.05 \mathrm{eV})$. In DFT for superconductors, calculations of $T_{c}$ (which is of the order of $0.001 \mathrm{eV}$ in general) are done simultaneously with those for the normalstate properties. This implies that the errors anticipated for $T_{c}$ would be very large compared to $T_{c}$ itself.

We should also point out that the present form for $F_{x c}[n, \chi]$ is useless to discuss the electronic mechanisms like the plasmon and the spin-fluctuation ones, prompting us to improve on the approximate form for $F_{x c}[n, \chi]$. Very recently, a limited improvement on $F_{x c}[n, \chi]$ was made by the inclusion of the contribution from plasmons, leading to better agreement with experiment for $T_{c} 66,67$.

Apart from the functional form, there are also several problems in the fundamental theory; for example, it is by no means clear whether the second-functional derivative of $F_{x c}[n, \chi]$ is a well-defined quantity or not, in just the same way as we have already experienced in the energygap problem $68-\underline{70}$ in semiconductors and insulators.

\section{EXPERIMENT ON SUPERCONDUCTIVITY IN GICS}

From this section, let us get back to the review on superconductivity in GICs. As briefly mentioned in Sec. 1, the history of the researches on this issue extends more than four decades. In 1965, the first report of superconductivity was made for $\mathrm{KC}_{8}, \mathrm{RbC}_{8}$, and $\mathrm{CsC}_{8}{ }^{5}$, in which $T_{c}$ was not reliably determined; it depended very much on samples. Subsequent works $-6,10,71-74$ confirmed the occurrence of superconductivity in $\mathrm{KC}_{8}$ with $T_{c}=0.15 \mathrm{~K}$, but superconductivity did not appear in $\mathrm{RbC}_{8}$ and $\mathrm{CsC}_{8}$ down to $0.09 \mathrm{~K}$ and $0.06 \mathrm{~K}$, respectively. Later works have found that $T_{c}$ is actually $26 \mathrm{mK}$ for $\mathrm{RbC}_{8} \frac{3}{\text {, but no su- }}$ perconductivity is found in either $\mathrm{LiC}_{6}$ or the secondor higher-stage alkali GICs, though the calculation of $T_{c}$ based on the McMillan's formula 30 predicted an observable value of $T_{c}$ even for $\mathrm{KC}_{24}{ }^{75,76}$. It seems that the usual first-principles calculation of $\alpha^{2} F(\omega)$ tends to provide an unrealistically large contribution from the intralayer high-energy carbon oscillations to $\lambda$. This unfavorable tendency in the calculation of $\lambda$ seems to prevail even in $\mathrm{CaC}_{6} 24$.

The anisotropy of the critical magnetic field $H_{c 2}$ was also a matter of interest, drawing attention of both experimentalists $8,10,12,74,77$ and theorists 78,79 . Note that the gap function $\Delta_{p}$ defined in Eq. (4) has nothing to do with the anisotropic behavior of $H_{c 2}$, though in developing a phenomenological theory ${ }^{78,79}$, some critical comments were made on the results of $\Delta_{p} \underline{\underline{ }}$ with the assumption that the anisotropy in $H_{c 2}$ should reflect on $\Delta_{p}$.

In search of higher $T_{c}$, many attempts have been made to synthesize new GIC superconductors such as $\mathrm{NaC}_{2}\left(T_{c}=5 \mathrm{~K}\right)^{\frac{81}{2}}, \operatorname{LiC}_{2}\left(T_{c}=1.9 \mathrm{~K}\right)^{14}$, and alkali- 
metal amalgams like $\mathrm{KHgC}_{4}\left(T_{c}=0.73 \mathrm{~K}\right)$ and $\mathrm{KHgC}_{8}$ $\left(T_{c}=1.90 \mathrm{~K}\right) 9,10,72,73,82,83$, but a larger enhancement of $T_{c}$ was not achieved until $\mathrm{CaC}_{6}$ was found in 2005 with $T_{c}=11.5 \mathrm{~K}^{15}$. Subsequently, many works have been done on alkaline-earth GIC superconductors $16,18,20,21,27,84-87$, but no one has ever succeeded in synthesizing a new GIC with $T_{c}$ larger than $15.4 \mathrm{~K}$ which was observed in $\mathrm{CaC}_{6}$ under pressures ${ }^{17}$. Thus some new idea seems to be needed to further enhance $T_{c}$. The present author hopes that the suggestions given in Sec. 8 help experimentalists synthesize a new GIC superconductor with $T_{c}$ much higher than $10 \mathrm{~K}$.

\section{STANDARD MODEL FOR SUPERCONDUCTIVITY IN GICS}

\section{A. Characteristic Features of the System}

Basically because GICs are not recognized as stronglycorrelated systems, the usual $a b$ initio self-consistent band-structure calculation is very useful in elucidating the important features of the electronic structures of GICs in the normal state. According to such calculations, it is found that there is no essential qualitative difference between alkali and alkaline-earth GICs (see Fig. 3). The main common features among these GICs may be summarized in the following way:
(a)Band structure of $\mathrm{CaC}_{6}$

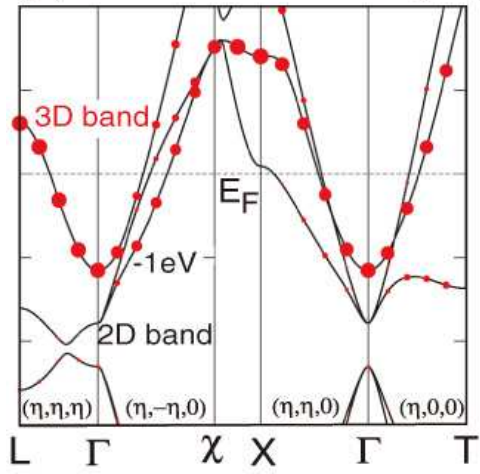

(b)Fermi surface of $\mathrm{KC}_{8}$

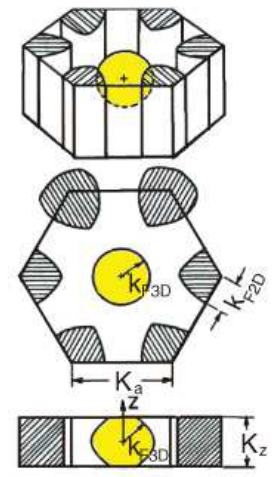

FIG. 3: (a)Band structure of $\mathrm{CaC}_{6}{ }^{24}$. (b)Fermi surface of $\mathrm{KC}_{8}{ }^{92}$. Both materials are characterized by the common feature that the electronic system is composed of the $2 \mathrm{D} \pi$ bands of graphite and the 3D interlayer band.

a) In $M \mathrm{C}_{x}$, each intercalant metal atom acts as a donor and changes from a neutral atom $M$ to an ion $M^{Z+}$ with valence $Z$.

b) The valence electrons released from $M$ will transfer either to the graphite $\pi$ bands or the threedimensional (3D) band composed of the intercalant orbitals and the graphite interlayer states ${ }^{88}-90$. We shall define the factor $f$ as the branching ratio between these two kinds of bands. Namely, $Z f$ and
$Z(1-f)$ electrons will go to the $\pi$ and the $3 \mathrm{D}$ bands, respectively.

c) The electrons in the graphite $\pi$ bands are characterized by the two-dimensional (2D) motion with a linear dispersion relation (known as a Dirac cone in the case of graphene) on the graphite layer.

d) The dispersion relation of the graphite interlayer band is very similar to that of the $3 \mathrm{D}$ free-electron gas, folded into the Brillouin zone of the graphite 23 . Thus its energy level is very high above the Fermi level in the graphite, because the amplitude of the wavefunction for this band is small on the carbon atoms. In $M \mathrm{C}_{x}$, on the other hand, the cation $M^{Z+}$ is located in the interlayer position where the amplitude of the wavefunctions is large, lowering the energy level of the interlayer band below the Fermi level. The dispersion of the interlayer band is modified from that of the free-electron gas because of the hybridization with the orbitals associated with $M$, but generally it is well approximated by $\varepsilon_{\boldsymbol{p}}=\boldsymbol{p}^{2} / 2 m^{*}-E_{\mathrm{F}}$ with an appropriate choice of the effective band mass $m^{*}$ and the Fermi energy $E_{\mathrm{F}}$. Here the value of $m^{*}$ depends on $M$; in alkali GICs, the hybridization occurs with s-orbitals, allowing us to consider that $m^{*}=m_{e}$, while in alkaline-earth GICs, the hybridization with d-orbitals contributes much, leading to $m^{*} \approx 3 m_{e}$ in both $\mathrm{CaC}_{6}$ and $\mathrm{YbC}_{6}$, as revealed by the bandstructure calculation 22,24 .

e) The value of $f$, which determins the branching ratio $Z f: Z(1-f)$, can be obtained by the self-consistent band-structure calculation. In $\mathrm{KC}_{8}$, for example, it is known that $f$ is around $0.6^{91}$. On the other hand, $f$ is about $0.16^{24}$ in $\mathrm{CaC}_{6}$, making the electron density in the $3 \mathrm{D}$ band $n$ increase very much. This increase in $n$ is easily understood by the fact that the energy level of the interlayer band is much lower with $\mathrm{Ca}^{2+}$ than with $\mathrm{K}^{+}$. The concrete numbers for $n$ are $3.5 \times 10^{21} \mathrm{~cm}^{-3}$ and $2.4 \times 10^{22} \mathrm{~cm}^{-3}$ for $\mathrm{KC}_{8}$ and $\mathrm{CaC}_{6}$, respectively, in which the difference in both $d$ and $x$ is also taking into account.

f) As inferred from experiments $2,23,80$ and also from the comparison of $T_{c}$ calculated for each band ${ }^{80}$, it has been concluded that only the 3D interlayer band is responsible for superconductivity. Note that $\mathrm{LiC}_{6}$ does not exhibit superconductivity because no carriers are present in the $3 \mathrm{D}$ interlayer band, although the properties of $\mathrm{LiC}_{6}$ are generally very similar to those of other superconducting GICs in the normal state.

\section{B. Microscopic Model for Superconductivity}

With these common features in mind, we can think of a simple model for the GIC superconductors, which 
is schematically shown in Fig. 4(a). Actually, exactly the same model was proposed in as early as 1982 by the present author for describing superconductivity in alkali GICs 80 .

(a)Model for $M C_{X}(x=2,6,8)$ :
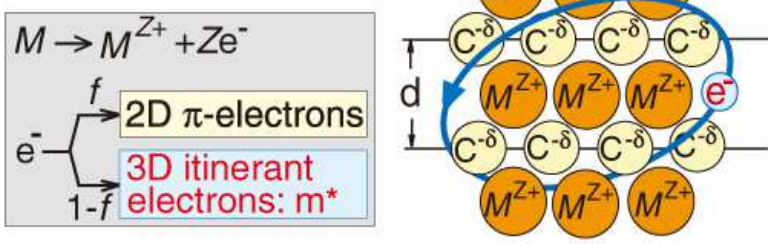

$(\delta=Z f / x)$

(b)Effective electron-electron interaction: $V(q, i \Omega)$

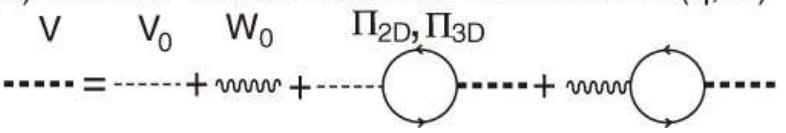

FIG. 4: (a)Simplified model to represent $M \mathrm{C}_{x}$ superconductors. We consider the attraction between the $3 \mathrm{D}$ electrons in the interlayer band induced by polar-coupled charge fluctuations of the cation $M^{Z+}$ and the anion $\mathrm{C}^{-\delta}$. (b)Diagrammatic representation of the equation in the RPA to calculate the effective electron-electron interaction $V\left(\boldsymbol{p}-p^{\prime}, i \Omega\right)$, which will be substituted in Eq. (6) to evaluate the kernel of the gap equation.

In order to give some idea about the mechanism to induce an attraction between 3D electrons in this model, let us imagine how each conducting 3D electron sees the charge distribution of the system. First of all, there are positively charged metallic ions $M^{Z+}$ with its density $n_{M}$, given by $n_{M}=4 / 3 \sqrt{3} a^{2} d x$, where $a$ is the bond length between $\mathrm{C}$ atoms on the graphite layer (which is $1.419 \AA)$. Note that with use of this $n_{M}$, the density of the $3 \mathrm{D}$ electrons $n$ is given by $(1-f) Z n_{M}$. There are also negatively charged carbon ions $\mathrm{C}^{-\delta}$ with the average charge of $\delta \equiv-f Z e / x$. Therefore the 3D electrons will feel a large electric field of the polarization wave coming from oscillations of $M^{Z+}$ and $\mathrm{C}^{-\delta}$ ions created by either out-of-phase optic or in-phase acoustic phonons.

We shall consider the coupling of those phonons with the 3D electrons in terms of the point-charge model, allowing us to write the phonon-exchange polar-coupled interaction $W_{0}(\boldsymbol{q}, \omega)$ for the scattering of the $3 \mathrm{D}$ electrons with momentum- and energy-transfers of $\boldsymbol{q}$ and $\omega$ as

$$
\begin{aligned}
W_{0}(\boldsymbol{q}, \omega)= & V_{0}(\boldsymbol{q}) \frac{\omega_{p}^{2}(1-f)^{2}}{\omega^{2}-\omega_{\mathrm{LA}}(\boldsymbol{q})^{2}} \\
& +V_{0}(\boldsymbol{q}) \frac{\bar{\omega}_{p}^{2}\left(\bar{M} / M_{M}+f \bar{M} / x M_{C}\right)^{2}}{\omega^{2}-\omega_{\mathrm{LO}}(\boldsymbol{q})^{2}},
\end{aligned}
$$

with $\omega_{p}$ and $\bar{\omega}_{p}$ defined, respectively, as

$$
\omega_{p}=\sqrt{\frac{4 \pi e^{2} Z^{2} n_{M}}{M_{M}+x M_{C}}} \text { and } \bar{\omega}_{p}=\sqrt{\frac{4 \pi e^{2} Z^{2} n_{M}}{\bar{M}}},
$$

where $M_{M}$ and $M_{C}$ are, respectively, the atomic masses of $M$ and $\mathrm{C}, \bar{M}\left(=M_{M} x M_{C} /\left(M_{M}+x M_{C}\right)\right)$ is the reduced mass of $M \mathrm{C}_{x}, \omega_{\mathrm{LO}}(\boldsymbol{q})$ and $\omega_{\mathrm{LA}}(\boldsymbol{q})$ are the energies of LO- and LA-phonons, respectively, and $V_{0}(\boldsymbol{q})$ is the bare Coulomb interaction $4 \pi e^{2} / \boldsymbol{q}^{2}$. (The subscript 0 indicates that it is the bare interaction to be screened by both 2D and 3D mobile electrons.)

Owing to the coupling with valence electrons, both $\omega_{\mathrm{LO}}(\boldsymbol{q})$ and $\omega_{\mathrm{LA}}(\boldsymbol{q})$ depends on $f$, but the $f$-dependence is not important, if we write the phonon-mediated interaction in terms of the corresponding transverse phonon energies, $\omega_{\mathrm{TO}}(\boldsymbol{q})$ and $\omega_{\mathrm{TA}}(\boldsymbol{q})$. Thus we specify the phonon energies in terms of $\omega_{\mathrm{TO}}(\boldsymbol{q})$ and $\omega_{\mathrm{TA}}(\boldsymbol{q})$. In actual calculations, we assume that $\omega_{\mathrm{TO}}(\boldsymbol{q})=\omega_{t}(=$ constant) and $\omega_{\mathrm{TA}}(\boldsymbol{q})=c_{t}|\boldsymbol{q}|$ with $\omega_{t}$ of the order of $150 \mathrm{~K}$ and $c_{t}$ of the order of $10^{5} \mathrm{~cm} \mathrm{~s}^{-1}$ for the oscillation perpendicular to the graphite plane.

\section{Calculation of $T_{c}$ for Alkali-Doped GICs}

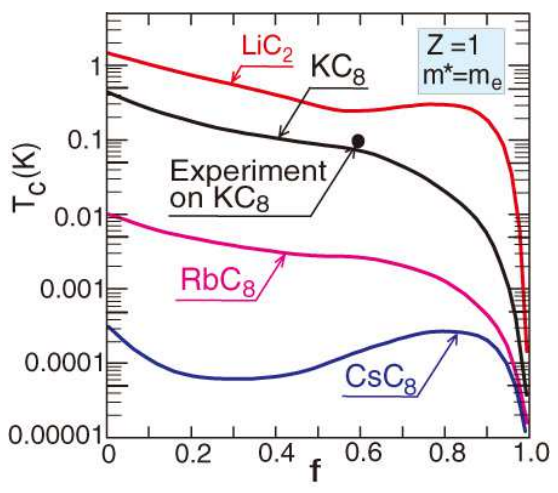

FIG. 5: Calculated results for $T_{c}$ as a function of the branching ratio $f$ for alkali GIC superconductors in which $Z=1$ and $m^{*}=m_{e}$.

By combining this polar-phonon-mediated attractive interaction $W_{0}(\boldsymbol{q}, \omega)$ with the bare Coulomb interaction between electrons $V_{0}(\boldsymbol{q})$ on the same footing and considering the polarization effects of both $2 \mathrm{D}$ and $3 \mathrm{D}$ electrons, we faithfully calculate $V(\boldsymbol{q}, \omega)$ the effective interaction

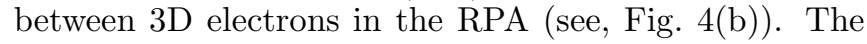
obtained $V(\boldsymbol{q}, \omega)$ is put into the kernel, Eq. (6) , of the gap equation (4) to obtain $T_{c}$ from first principles. The calculated results for $T_{c}$ in alkali GICs are plotted as a function of $f$ in Fig. 5 to find that the overall magnitude of $T_{c}$ is in the range of $0.1-0.01 \mathrm{~K}$ for $f \approx 0.5$, in good agreement with experiment. Note that smaller values of $T_{c}$ are obtained for heavier alkali atoms because of the smaller couplings as characterized by both $\omega_{p}$ and $\bar{\omega}_{p}$. This success indicates that the present simple model applies well at least to alkali GIC superconductors. 


\section{SUPERCONDUCTIVITY IN ALKALINE-EARTH GICS}

A. $\mathrm{CaC}_{6}$

Now let us consider alkaline-earth GIC superconductors. We shall investigate them by adopting the same simple model with using exactly the same calculation code developed in 1982 in order to see whether the model and therefore the piture on the mechanism of superconductivity successfully applied to alkali GIC superconductors can also be relevant to these newly-synthesized superconductors or not $\underline{28,29}$. The parameters specifying the model will be changed in the following way, if $\mathrm{CaC}_{6}$ is considered instead of $\mathrm{KC}_{8}$ :

a) Because the valence $Z$ changes from monvalence to divalence, the atractive interaction $W_{0}$, which is in proportion to $Z^{2}$, increases by four times.

b) The interlayer distance $d$ decreases from $5.42 \AA$ to $4.524 \AA$, so that the $3 \mathrm{D}$ electron density $n$ increases.

c) The factor $f$ to determine the branching ratio decreases from about 0.6 to 0.16 .

d) The effective band mass for the 3D interlayer band $m^{*}$ increases from $m_{e}$ to about $3 m_{e}$.

e) The atomic number of the ion $A$ hardly changes from 39.1 to 40.1 .

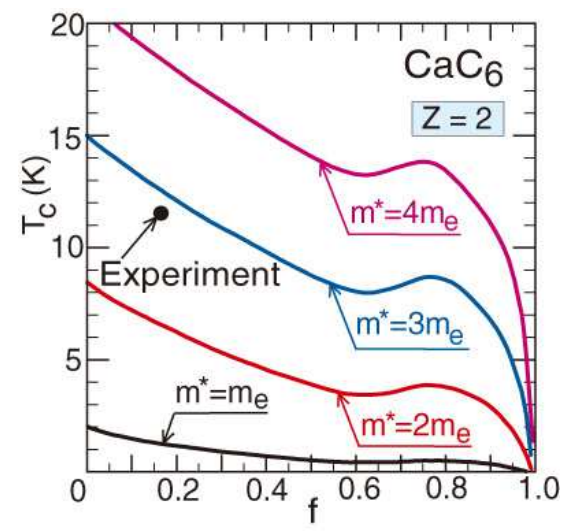

FIG. 6: Calculated $T_{c}$ as a function of $f$ for $m^{*}$ in the range of $m_{e}-4 m_{e}$ with other parameters suitably chosen for $\mathrm{CaC}_{6}$. The experimental result is reproduced well, if we choose $m^{*} \approx$ $3 m_{e}$.

With paying attention to these changes of the parameters, we have calculated $T_{c}$ for $\mathrm{CaC}_{6}$ as a function of $f$. The results are plotted in Fig. 6, from which we can learn the following points:

1) Overall, $T_{c}$ becomes higher for smaller $f$. This can be understood by the fact that the screening effect due to the 2D $\pi$ electrons, which makes the polarcoupled interaction weak, becomes smaller with the decrease of $f$.

2) The enhancement of $T_{c}$ by about one order is brought about by doubling $Z$, if $m^{*}$ is kept to be the same value.

3) The enhancement of $T_{c}$ by about one order is also brought about by tripling $m^{*}$ from $m_{e}$ to $3 m_{e}$, if $Z$ is taken as $Z=2$.

Based on these observations, we can conclude that the enhancement of $T_{c}$ in $\mathrm{CaC}_{6}$ by about a hundred times from that in $\mathrm{KC}_{8}$ is brought about by the combined effects of doubling $Z$ and tripling $m^{*}$. In this respect, the value of $m^{*}$ is very important. Appropriateness of $m^{*} \approx 3 m_{e}$ is confirmed not only from the band-structure calculations ${ }^{22,24}$ but also from the measurement of the electronic specfic heat $\underline{20}$ compared with the corresponding one for $\mathrm{KC}_{8}{ }^{93}$.

\section{B. Other alkaline-earth GICs}

Similar calculations are done for other alkaline-earth GIC superconductors as shown in Fig. 7 in which $m^{*}$ is determined so as to reproduce $E_{\mathrm{F}}$ supplied by the bandstructure calculation. We see that although we give $T_{c}$ a little larger than the experimental one for $\mathrm{SrC}_{6}$, overall good agreement is obtained between theory and experiment, implying that our simple model may be regarded as the standard one for describing the mechanism of superconductivity in GICs.

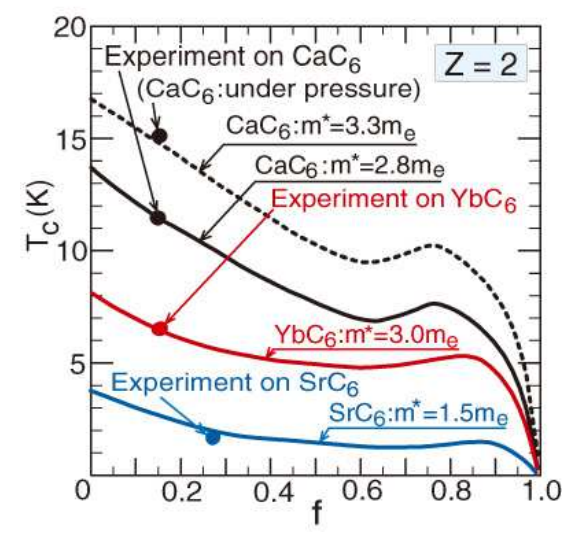

FIG. 7: Calculated $T_{c}$ as a function of $f$ for alkaline-earth GICs with $m^{*}$ determined so as to reproduce $E_{\mathrm{F}}$ provided by the band-structure calculation.

Here a note will be added to the case of $\mathrm{YbC}_{6}$; the basic parameters such as $Z, f$, and $m^{*}$ for $\mathrm{YbC}_{6}$ are about the same as those for $\mathrm{CaC}_{6}$, according to the bandstructure calculation. The only big change can be seen in 
the atomic mass; Yb (in which $A=173.0$ ) is much heavier than that of Ca by about four times, indicating weaker couplings between electrons and polar phonons as just in the case of comparison between $\mathrm{KC}_{8}$ and $\mathrm{CsC}_{8}$. In fact, $T_{c}$ for $\mathrm{YbC}_{6}$ becomes about one half of the corresponding result for $\mathrm{CaC}_{6}$, which agrees well with experiment. One way to understand this difference is to regard it as an isotope effect with $\alpha \approx 0.5^{22}$.

\section{C. $\mathrm{BaC}_{6}$}

The experimental results for $T_{c}$ in the alkaline-earth GICs treated in Fig. 7 are also well reproduced by the the conventional Eliashberg theory in which the McMillan's formula for $T_{c}$ is employed with use of the electronphonon coupling constant $\lambda$, the average phonon energy $\omega_{0}$, and the Coulomb pseudopotential $\mu^{*}$ with its conventional value of $\mu^{*}=0.14$. The two parameters, $\lambda$ and $\omega_{0}$, are determined by the first-principles calculation of the Eliashberg function $\alpha^{2} F(\omega)^{94}$.

This success of the Eliashberg theory is, however, limited; the same theory predicts that $\mathrm{BaC}_{6}$ superconducts at $T_{c}=0.23 \mathrm{~K}$, but it turns out that superconductivity does not appear at least down to $80 \mathrm{mK}^{95}$. In search of the reason for this discrepancy between theory and experiment, the phonon structure is extensively studied in comparison with the case of $\mathrm{CaC}_{6}{ }^{96.97}$, but no persuasive reason has been found. In the present author's view, this failure is directly connected with the problem of obtaining an unrealistically large contribution from the intralayer high-energy carbon oscillations to $\alpha^{2} F(\omega)$, as mentioned in the first paragraph in Sec. V. In fact, $\omega_{0}=22.44 \mathrm{meV}$ is obtained for $\mathrm{BaC}_{6}{ }^{94}$, which is much higher than the energy of Ba oscillations $(\approx 8 \mathrm{meV})$, indicating that the carbon modes are responsible for the unsuccessful prediction of $T_{c}=0.23 \mathrm{~K}$ in the conventional Eliashberg theory.

Very recently $\mathrm{BaC}_{6}$ is discovered to exhibit superconductivity with $T_{c}=65 \mathrm{mK}^{19}$. In the framework of the standard model, $m^{*}, f$, and $Z$ are the important parameters to be determined by the band-structure calculation, from which we see that we may take $Z=2$ and $f$ in the range of $0.1-0.3$, the same situation as those in $\mathrm{CaC}_{6}$ and $\mathrm{SrC}_{6}$. As for $m^{*}$, it becomes smaller than $3 m_{e}$, because the interlayer 3D band of graphite is hybidized with the more itinerant $5 \mathrm{~d}$ orbitals in $\mathrm{BaC}_{6}$ compared with the $3 \mathrm{~d}$ ones in $\mathrm{CaC}_{6}$; if we compare the dispersion relation for the $3 \mathrm{D}$ band along $\Gamma \chi$ direction for $\mathrm{BaC}_{6}$ as shown in Fig. 8(a) with that for $\mathrm{CaC}_{6}$ given in Fig. 31(a), we find that $m^{*} \approx 1.9 m_{e}$. In addition, the $3 \mathrm{D}$ band at $L$ point is located below the Fermi level due to the shorter Brilloiun zone (or equivalently the longer lattice constant) for $\mathrm{BaC}_{6}$, indicating that some portion of the otherwise spherical Fermi surface is truncated or missing, as seen in Fig. 8(b) which displays the Fermi surfaces for the 3D interlayer bands in $\mathrm{CaC}_{6}, \mathrm{SrC}_{6}$, and $\mathrm{BaC}_{6}$. Because of this truncation or missing, the virtual multiple scatter- ings to form the Cooper pairs are restricted, so that $T_{c}$ will be suppressed from the value obtained in the standard model. Note that, though its size is much smaller, this truncation or missing is also seen in $\mathrm{SrC}_{6}$ and thus the reduction of $T_{c}$ in experiment for $\mathrm{SrC}_{6}$ from that predicted in the standard model may be ascribed to this effect. (a) Band structure
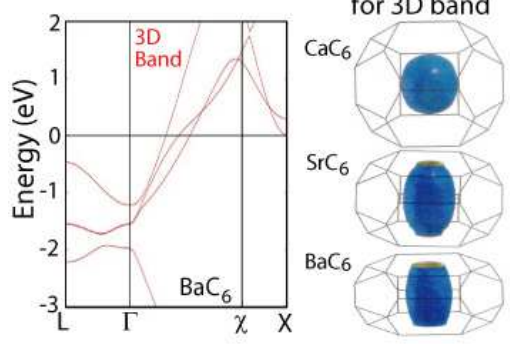

(b) Fermi surface for $3 \mathrm{D}$ band (c) Effect of $\varepsilon_{\infty}$ on $T_{c}$

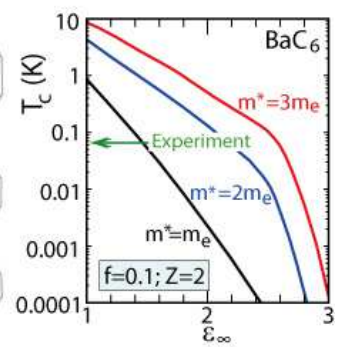

FIG. 8: (a) Band structure of $\mathrm{BaC}_{6}$. (b) The Fermi surfaces for the $3 \mathrm{D}$ interlayer bands in $\mathrm{CaC}_{6}, \mathrm{SrC}_{6}$, and $\mathrm{BaC}_{6}{ }^{94}$. (c) Calculated $T_{c}$ as a function of the optic dielectric constant $\varepsilon_{\infty}$ for $\mathrm{BaC}_{6}$ with $m^{*}$ in the range $m_{e}-3 m_{e}$. Note that $T_{c}$ is $65 \mathrm{mK}$ experimentally ${ }^{19}$.

This missing of the sperical Fermi surface implies the reduction of the density of states for the $3 \mathrm{D}$ interlayer band at the Fermi level and therefore it might be effectively taken into account by the reduction of $m^{*}$ from that in the band-structure calculation. Probably $m^{*} \approx 1.5 m_{e}$ will be a reasonable choice. With this idea in mind, we have calculated $T_{c}$ for $\mathrm{BaC}_{6}$ with $m^{*}$ in the range $m_{e}-2 m_{e}$ to find that, irrespective of $f$ taken in the range of $0.1-0.5$, the obtained $T_{c}$ is always larger than $0.1 \mathrm{~K}$, which is about the same as that in the Eliashberg theory but is much higher than the experimental value. Thus we need to look for another crucial parameter in the standard model to explain the experimental value of $T_{c}$.

Basically, the standard model assumes the polarcoupling phonon mechanism of superconductivity in which, in general, the effect of the optic dielectric constant $\varepsilon_{\infty}$ should be included in the theory and can be treated by changing $e^{2}$ into $e^{2} / \varepsilon_{\infty}$ in Eqs. (8) and (9). Physically $\varepsilon_{\infty}$ is determined by the magnitude of core polarization of constituent atoms or ions. For light atoms like carbon, the core polarization is negligibly small and thus we may well take $\varepsilon_{\infty}$ as unity. Even for $\mathrm{Ca}^{2+}$, its polarizability is about 3.2 in atomic units ${ }^{98}$, leading to $\varepsilon_{\infty}=1.07$. For heavy atoms, however, it can never be neglected; for $\mathrm{Ba}$, the polarizabilities are, respectively, 124 and 10.5 for $\mathrm{Ba}^{+}$and $\mathrm{Ba}^{2+}$, which correspond, respectively, to $\varepsilon_{\infty}=3.8$ and 1.24. By combining these numbers for $\varepsilon_{\infty}$ with the fact that the $3 \mathrm{D}$ interlayer band is completely occupied in the $\Gamma-L$ direction, making some portion of the released 6 s electrons actually localize near the $\mathrm{Ba}^{2+}$ site, leading effectively to the state of $\mathrm{Ba}^{(2-\delta)+}$, we may assume that the effective value for $\varepsilon_{\infty}$ is in the 
range of $1.5-2.0$. Then, as we can see in Fig. 8)(c), $T_{c}$ obtained in the standard model with $m^{*} \approx 1.5 m_{e}$ fits well with the experimental one.

\section{PREDICTION OF THE OPTIMUM $T_{c}$ IN GICS}

As we have seen so far, our standard model could have predicted $T_{c}=11.5 \mathrm{~K}$ for $\mathrm{CaC}_{6}$ in 1982 and it is judged that its predictive power is very high. Incidentally, the author did not perform the calculation of $T_{c}$ for $\mathrm{CaC}_{6}$ at that time, partly because he did not know a possibility to synthesize such GICs, but mostly because the calculation cost was extremely high in those days; a rough estimate shows that there is acceleration in computers by at least a millon times in the past three decades. This huge improvement on computational environments is surely a boost to making such first-principles calculations of $T_{c}$ as reviewed in Secs. 3 and 4.

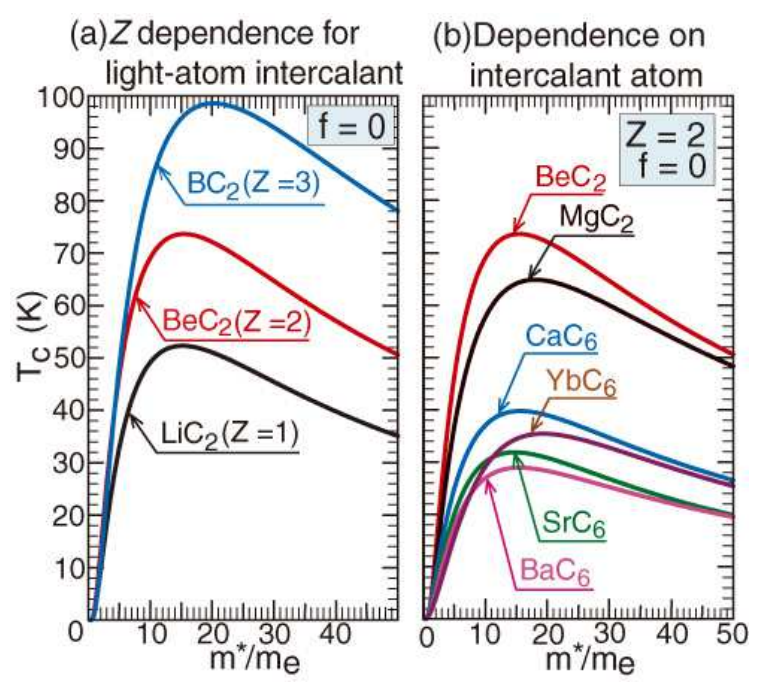

FIG. 9: Prediction of $T_{c}$ as a function of $m^{*}$ for various GICs in pursuit of optimum $T_{c}$. We assume the fractional factor $f=0$.

In any way, encouraged by this success in reproducing $T_{c}$ in alkaline-earth GICs, we have explored the optimum $T_{c}$ in the whole family of GICs by widely changing various parameters involved in the microscopic Hamiltonian. Examples of the calculated results of $T_{c}$ are shown in Fig. 9(a) and (b), in which $f$ is fixed to zero, the optimum condition to raise $T_{c}$, and $d$ is tentatively taken as $4.0 \AA$. From this exploration, we find that the most important parameter to enhance $T_{c}$ is $m^{*}$. In particular, we need $m^{*}$ larger than at least $2 m_{e}$ to obtain $T_{c}$ over $10 \mathrm{~K}$, irrespective of any choice of other parameters, and $T_{c}$ is optimized at $m^{*}$ near $15 m_{e}$. The optimized $T_{c}$ depends rather strongly on the parameters to control the polarcoupling strength such as $Z$ and the atomic mass $A$; if we choose a trivalent light atom such as boron to make $\omega_{t}$ large, the optimum $T_{c}$ is about $100 \mathrm{~K}$, but the problem about the light atoms is that $m^{*}$ will never become heavy due to the absence of either $\mathrm{d}$ or $\mathrm{f}$ electrons. Therefore we do not expect that $T_{c}$ would become much larger than $10 \mathrm{~K}$, even if $\mathrm{BeC}_{2}$ or $\mathrm{BC}_{2}$ were synthesized. From this perspective, it will be much better to intercalate $\mathrm{Ti}$ or $\mathrm{V}$, rather than Be or B. Taking all these points into account, we suggest synthesizing three-element GICs providing a heavy $3 \mathrm{D}$ electron system by the introduction of heavy atoms into a light-atom polar-crystal environment.

\section{CONCLUSION}

In this chapter, by taking account of the common features elucidated by both the band-structure calculation and the various measurements on the normal-state properties, we have constructed the standard model pertinent for the description of the mechanism of superconducitity in metal GICs and then made first-principles calculations of $T_{c}$ in the $G_{0} W_{0}$ scheme, directly from the microscopic Hamiltonian representing the standard model. With suitably choosing the parameters in the microscopic Hamiltonian, we have found surprisingly good agreement between theory and experiment for both alkali and alkaline-earth GICs, in spite of the fact that $T_{c}$ varies more than three orders of magnitude. In this way, we have clarified that superconductivity in metal GICs can be understood by the picture that the $3 \mathrm{D}$ electrons in the interlayer band supplied by the ionization of metals feel the attractive interaction induced by the virtual exchange of the polarcoupled phonons of the metal ions. We have also predicted a further enhancement of $T_{c}$ well beyond $10 \mathrm{~K}$ with giving some suggestions to realize such superconductors in the family of GICs.

By first-principles we usually mean the calculations based on not the model but the first-principles Hamiltonian. Thus it might be considered as inappropriate to call the present $G_{0} W_{0}$ scheme first-principles, but it is not an easy task to specify the key parameters to control $T_{c}$ by just implementing the calculations based on the first-principles Hamiltonian. We can identify the importance of the parameters, $m^{*}$ and $Z$, only through the calculations based on the model Hamiltonian, leading to the better and unambiguous understanding of the mechanism of superconductivity without involved too much into the very details of each system which sometimes obscure the essence in first-principles approaches. Besides, because of the errors involved in the numerical calculations of normal-state properties as mentioned in Sec. 4, more accurate results of $T_{c}$ will be obtained by way of a suitable model Hamiltonian rather than directly from the first-principles one.

As a project in the future, it would be important to construct a more powerful scheme for the first-principles calculation of $T_{c}$ by the combination of the schemes in Secs. 3 and 4 , based on which we may make more detailed suggestions to synthesize GIC superconductors with $T_{c}$ 
much larger than $10 \mathrm{~K}$.

* Email: takada@issp.u-tokyo.ac.jp; published in Reference Module in Materials Science and Materials Engineering (Saleem Hashmi; editor-in-chief), Oxford, Elsevier, 2016; DOI:10.1016/B978-0-12-803581-8.00774-8

1 J. EDFischer and TDEDThompson, Phys. Today 31, Issue 7, 36i1978).

2 H. Kamimura, Phys. Today 40, Issue 12, 64 (1987).

${ }^{3}$ H. Zabel and S. A. Solin (Eds.), Graphite Intercalation Compounds II, Springer-Verlag, 1992.

4 M. S. Dresselhaus and G. Dresselhaus, Adv. Phys. 51, 1 (2002).

5 N. B. Hannay, T. H. Geballe, B. T. Matthias, K. Andres, P. Schmidt, and D. MacNair, Phys. Rev. Lett. 14, 225 (1965).

6 Y. Koike, H. Suematsu, K. Higuchi, and S. Tanuma, Solid State Commun. 27, 623 (1978).

7 M. Kobayashi and I. Tsujikawa, J. Phys. Soc. Jpn. 46, 1945 (1979).

8 Y. Koike, H. Suematsu, K. Higuchi, and S. Tanuma, Physica B 99, 503 (1980).

9 M. G. Alexander, D. P. Goshorn, D. Guerard, P. Lagrange, M. El Makrini, and D. G. Onn, Synth. Met. 2, 203 (1980).

10 Y. Iye and S. Tanuma, Phys. Rev. B 25, 4583 (1982).

11 I. T. Belash, O.V. Zharikov, and A.V. Pal'nichenko, Synth. Met. 34, 455 (1989).

12 M. S. Dresselhaus, A. Chaiken, and G. Dresselhaus, Synth. Met. 34, 449 (1989).

13 I. T. Belash, A. D. Bronnikov, O.V. Zharikov, and A.V. Pal'nichenko, Synth. Met. 36, 283 (1990).

14 I. T. Belash, A. D. Bronnikov, O.V. Zharikov, and A.V. Pal'nichenko, Solid State Commun. 69, 921 (1989).

15 T. E. Weller, M. Ellerby, A. S. Saxena, R. P. Smith, and N. T. Skipper, Nature Phys. 1, 39 (2005).

16 N. Emery, C. Heérold, M. d'Astuto, V. Garcia, C. Bellin, J. F. Marêché, P. Lagrange, and G. Loupias, Phys. Rev. Lett. 95, 087003 (2005).

17 A. Gauzzi, S. Takashima, N. Takeshita, C. Terakura, H. Takagi, N. Emery, C. Hérold, P. Lagrange, and G. Loupias, Phys. Rev. Lett. 98, 067002 (2007).

18 J. S. Kim, L. Boeri, J. R. O'Brien, F. S. Razavi, and R. K. Kremer, Phys. Rev. Lett. 99, 027001 (2007).

19 S. Heguri, N. Kawade, T. Fujisawa, A. Yamaguchi, A. Sumiyama, K. Tanigaki, and M. Kobayashi, Phys. Rev. Lett. 114, 247201 (2015).

20 J. S. Kim, R. K. Kremer, L. Boeri, and F. S. Razavi, Phys. Rev. Lett. 96, 217002 (2006).

21 C. Kurter, L. Ozyuzer, D. Mazur, J. F. Zasadzinski, D. Rosenmann, H. Claus, D. G. Hinks, and K. E. Gray, Phys. Rev. B 76, 220502 (2007).

${ }^{22}$ I. I. Mazin, Phys. Rev. Lett. 95, 227001 (2005).

23 G. Csanyi, P. B. Littlewood, A. H. Nevidomskyy, C. J. Pickard, and B. D. Simons, Nature Phys. 1, 42 (2005).

24 M. Calandra and F. Mauri, Phys. Rev. Lett. 95, 237002 (2005).

25 M. Calandra and F. Mauri, Phys. Rev. B 74, 094507 (2006).

26 A. Sanna, G. Profeta, A. Floris, A. Marini, E. K. U. Gross, and S. Massidda, Phys. Rev. B 75, 020511(R) (2007).
27 D. G. Hinks, D. Rosenmann, H. Claus, M. S. Bailey, and J. D. Jorgensen, Phys. Rev. B 75, 014509 (2007).

28 Y. Takada, J. Phys. Soc. Jpn. 78, 013703 (2009).

29 Y. Takada, J. Supercond. Nov. Magn. 22, 89 (2009).

30 W. L. McMillan, Phys. Rev. 167, 331 (1968).

31 P. B. Allen and R. C. Dynes, Phys. Rev. B 12, 905 (1975).

32 P. B. Allen and B. Mitrović, in Solid State Physics, edited by H. Ehrenreich, F. Seitz, and D. Turnbull, Vol. 37, p. 1 (Academic, New York, 1982).

33 J.P. Carbotte, Rev. Mod. Phys. 62, 1027 (1990).

${ }^{34}$ G. M. Eliashberg, Sov. Phys.-JETP 11, 696 (1960).

35 P. Morel and P. W. Anderson, Phys. Rev. 125, 1263 (1962).

36 K.-P. Bohnen, R. Heid, and B. renker, Phys. Rev. Lett. 86, 5771 (2001).

37 Y. Kong, O. V. Dolgov, O. Jepsen, and O. K. Andersen, Phys. Rev. B 64, 020501(R) (2001).

38 H. J. Choi, D. Roundy, H. Sun, M. L. Cohen, and S. G. Louie, Nature 418, 758 (2002).

39 H. J. Choi, D. Roundy, H. Sun, M. L. Cohen, and S. G. Louie, Phys. Rev. B 66, 020513(R) (2002).

40 Y. Takada, J. Phys. Soc. Jpn. 45, 786 (1978).

41 Y. Takada, Phys. Rev. B 47, 5202 (1993).

42 A. F. Hebard, M. J. Rosseinsky, R. C. Haddon, D. W. Murphy, S. H. Glarum, T. T. M. Palstra, A. P. Ramirez, and A. R. Kortan, Nature 350, 600 (1991).

43 Y. Takabayashi, A. Y. Ganin, P. Jeglič, D. Arčon, T. Takano, Y. Iwasa, Y. Ohishi, M. Takata, N. Takeshita, K. Prassides, and M. J. Rosseinsky, Science 323, 1585 (2009).

44 O. Gunnarsson, Rev. Mod. Phys. 69, 575 (1997).

45 Y. Takada and T. Hotta, Int. J. Mod. Phys. B 12, 3042 (1998).

46 Y. Takada, J. Phys. Chem. Solids 54, 1779 (1993).

47 We employ units in which $\hbar=k_{\mathrm{B}}=1$.

48 M. L. Cohen, Phys. Rev. 134 A511 (1964).

49 M. L. Cohen, in "Superconductivity", ed.R. D. Parks (Marcel Dekker, New York, 1969) Vol. 1, Chap. 12.

50 Y. Takada, J. Phys. Soc. Jpn. 49, 1267 (1980).

51 D. A. Kirzhnits, E. G. Maksimov, and D. I. Khomskii, J. Low Temp. Phys. 10, 79 (1973).

52 X. Lin, Z. Zhu, B. Fauqué, and K. Behnia, Phys. Rev. X 3, 021002 (2013).

53 X. Lin, G. Bridoux, A. Gourgout, G. Seyfarth, S. Krämer, M. Nardone, B. Fauqué, and K. Behnia, Phys. Rev. Lett. 112, 207002 (2014).

54 P. Hohenberg and W. Kohn, Phys. Rev. 136, 864 (1964).

55 W. Kohn and L. J. Sham, Phys. Rev. 140, A1133 (1965).

56 L. N. Oliveira, E. K. U. Gross, and W. Kohn, Phys. Rev. Lett. 60, 2430 (1988).

57 S. Kurth, M. Marques, M. Lüders, and E. K. U. Gross, Phys. Rev. Lett. 83, 2628 (1999).

58 P. G. de Gennes, Superconductivity of Metals and Alloys, (Benjamin, New York, 1966).

59 M. Lüders, M. A. L. Marques, N. N. Lathiotakis, A. Floris, G. Profeta, L. Fast, A. Continenza, S. Massidda, and E. K. U. Gross, Phys. Rev. B 72, 024545 (2005).

${ }^{60}$ M. A. L. Marques, M. Lüders, N. N. Lathiotakis, G. Profeta, A. Floris, L. Fast, A. Continenza, E. K. U. Gross, and 
S. Massidda, Phys. Rev. B 72, 024546 (2005).

61 A. Floris, G. Profeta, N. N. Lathiotakis, M. Lüders, M. A. L. Marques, C. Franchini, E. K. U. Gross, A. Continenza, and S. Massidda, Phys. Rev. Lett. 94, 037004 (2005).

62 G. Profeta, C. Franchini, N. N. Lathiotakis, A. Floris, A. Sanna, M. A. L. Marques, M. Lüders, S. Massidda, E. K. U. Gross, and A. Continenza, Phys. Rev. Lett. 96, 047003 (2006).

63 A. Sanna, C. Franchini, A. Floris, G. Profeta, N. N. Lathiotakis, M. Lüders, M. A. L. Marques, E. K. U. Gross, A. Continenza, and S. Massidda, Phys. Rev. B 73, 144512 (2006).

64 A. Floris, A. Sanna, S. Massidda, and E. K. U. Gross, Phys. Rev. B 75, 054508 (2007).

65 J. P. Perdew, K. Burke, and M. Ernzerhof, Phys. Rev. Lett. 77, 3865 (1996); ibid. 78, 1396 (1997) (E).

66 R. Akashi and R. Arita, Phys. Rev. Lett. 111, 057006 (2013).

67 R. Akashi and R. Arita, J. Phys. Soc. Jpn. 83, 061016 (2014).

68 J. P. Perdew and M. Levy, Phys. Rev. Lett. 51, 1884 (1983).

69 L. J. Sham and M. Schlüter, Phys. Rev. Lett. 51, 1888 (1983)

${ }^{70}$ L. J. Sham and M. Schlüter, Phys. Rev. B 32, 3883 (1985).

71 M. Kobayashi and I. Tsujikawa, Physica B 105, 439 (1981).

72 L. A. Pendrys, R. Wachnik, F. L. Vogel, P. Lagrange, G. Furdin, M. EI-Makrini, and A. Herold, Solid State Commun. 38, 677 (1981).

73 M. G. Alexander, D. P. Goshorn, D. Guerard, P. Lagrange, M. EI Makrini, and D. G. Onn, Solid State Commun. 38, 103 (1981).

74 A. Chaiken, M. S. Dresselhaus, T. P. Orlando, G. Dresselhaus, P.M. Tedrow, D. A. Neumann, and W. A. Kamitakahara, Phys. Rev. B 41, 71 (1990).

75 H. Kamimura, K. Nakao, T. Ohno and T. Inoshita, Physica B 99, 401 (1980).

76 T. Inoshita and H. Kamimura, Synthetic Metals 3, 223 (1981).

77 G. Roth, A. Chaiken, T. Enoki, N. C. Yeh, G. Dresselhaus, and P. M. Tedrow, Phys. Rev. B 32, 533 (1985).

78 R. A. Jishi, M. S. Dresselhaus, and A. Chaiken, Phys. Rev. B 44, 10248 (1991).

79 R. A. Jishi and M. S. Dresselhaus, Phys. Rev. B 45, 12465 (1992).
${ }^{80}$ Y. Takada, J. Phys. Soc. Jpn. 51, 63 (1982)

81 I.T. Belash, A.D. Bronnikov, O.V. Zharikov, A.V. Palnichenko, Solid State Commun. 64, 1445 (1987).

82 S. Tanuma, Physica B 105, 486 (1981).

${ }^{83}$ Y. Koike and S. Tanuma, J. Phys. Soc. Jpn. 50, 1964 (1981).

84 G. Lamura, M. Aurino, G. Cifariello, E. Di Gennaro, A. Andreone, N. Emery, C. Hérold, J.-F. Marêché, and P. Lagrange, Phys. Rev. Lett. 96, 107008 (2006).

85 K. Kadowaki, T. Nabemoto, T. Yamamoto, Physica C 460-462, 152 (2007).

${ }^{86}$ K. Sugawara, T. Sato, and T. Takahashi, Nature Phys. 5, 40 (2009).

87 T. Valla, J. Camacho, Z.-H. Pan, A. V. Fedorov, A. C. Walters, C. A. Howard, and M. Ellerby, Phys. Rev. Lett. 102, 107007 (2009).

88 M. Posternak, A. Baldereschi, A. J. Freeman, and E. Wimmer, Phys. Rev. Lett. 52, 863 (1984).

89 N. A. W. Holzwarth, S. G. Louie, and S. Rabii, Phys. Rev. B 30, 2219 (1984).

90 A. Koma, K. Miki, H. Suematsu, T. Ohno, and H. Kamimura, Phys. Rev. B 34, 2434 (1986).

91 T. Ohno, K. Nakao, and H. Kamimura, J. Phys. Soc. Jpn. 47, 1125 (1979).

92 G. Wang, W. R. Datars, and P. K. Ummat, Phys. Rev. B 44, 8294 (1991).

93 U. Mizutani, T. Kondow, and T. B. Massalski, Phys. Rev. B 17, 3165 (1978).

94 M. Calandra and F. Mauri, Phys. Rev. B 74, 094507 (2006).

95 S. Nakamae, A. Gauzzi, F. Ladieu, D. L'Hôte, N. Eméry, C. Hérold, J. F. Marêché, P. Lagrange, and G. Loupias, Solid State Commun. 145, 493 (2008).

96 M. d'Astuto, M. Calandra, N. Bendiab, G. Loupias, F. Mauri, S. Zhou, J. Graf, A. Lanzara, N. Emery, C. Hérold, P. Lagrange, D. Petitgrand, and M. Hoesch, Phys. Rev. B 81, 104519 (2010).

97 A. C. Walters, C. A. Howard, M. H. Upton, M. P. M. Dean, A. Alatas, B. M. Leu, M. Ellerby, D. F. McMorrow, J. P. Hill, M. Calandra, and F. Mauri, Phys. Rev. B 84, 014511 (2011).

98 J. Mitroy, M. S. Safronova, and C. W. Clark, J. Phys. B: At. Mol. Opt. Phys. 43, 202001 (2010). 